\title{
Investigating Game Mechanics that Target Players' Self-Control While Maintaining Engagement
}

\author{
Milad Soroush ${ }^{1,2}$ \\ Mark Hancock $^{1,2}$ \\ ${ }^{1}$ Management Sciences, ${ }^{2}$ The Games Institute \\ University of Waterloo, Waterloo, Canada \\ \{msoroush, mark.hancock\}@uwaterloo.ca
}

\author{
Vanessa K. Bohns ${ }^{3}$ \\ ${ }^{3}$ ILR School \\ Cornell University, Ithaca, NY, USA \\ vkb28@cornell.edu
}

\begin{abstract}
Whenever someone chooses to study instead of going to a party, or forgo dessert after dinner, that person is exercising self-control. Self-control is essential for achieving longterm goals, but isn't easy. Games present a compelling opportunity to engage in tasks that allow a player to exercise and improve self-control, and consequently provide data about a person's cognitive capacity to exert self-control. However, exercising self-control can be effortful and depleting, which makes incorporating it into a game design that maintains engagement and quality of experience a challenge. We present the design of game mechanics for exercising and improving self-control, and an initial study that effectively demonstrates that games can be designed to engage a broad level of self-control processes without negatively affecting player engagement and experience. Our results also show that player performance is connected to trait-level self-control. We discuss how (for example) players with low trait self-control can therefore be identified, and games intended to improve or exercise self-control can dynamically adapt to this information.
\end{abstract}

\section{CCS CONCEPTS}

- Human-centered computing Empirical studies in HCI

\section{Author Keywords}

Self-Control; Games; Self-Control Improvement; Cognitive Control.

\section{INTRODUCTION}

Self-control is a critical component of effective goal pursuit. It allows us to make better, more mindful choices, and is associated with affective well-being and happiness in life $[4,28]$. Self-control has been used as an umbrella term that encompasses a number of related constructs, such as selfregulation [4], delay of gratification [45], impulse control

(C) 2018 Copyright is held by the owner/author(s). Publication rights licensed to ACM. This is the author's version of the work. It is posted here for your personal use. Not for redistribution. The definitive Version of Record was published in Proceedings of the 2018 Annual Symposium on Computer-Human Interaction in Play Companion Extended Abstracts, http://dx.doi.org/10.1145/3242671.3242698

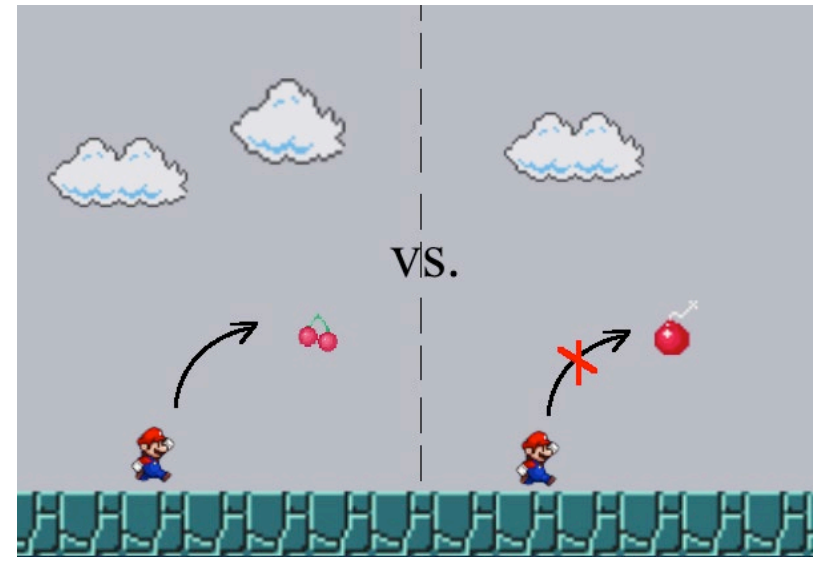

Figure 1. In Save the Garden, players exercise self-control by collecting fruit (left) and avoiding bombs (right). Players inhibit responses (e.g.) by jumping vs. not jumping.

[63], cognitive control and executive functions [16]. A key question for scientists is identifying factors that can help people to regulate their behaviour more effectively in order to achieve academic goals, create healthy habits, prevent unethical behaviour in the workplace, resist drug or alcohol consumption, avoid excessive use of media, et cetera.

The importance of measuring and improving self-control is made clear by remarkable findings on the effect self-control has on numerous positive outcomes in life. In one of the original studies of self-control, which focused specifically on the construct of delay of gratification, measured by the now well-known "Marshmallow Test", Mischel et al. [45] demonstrated that the ability of preschoolers to wait for a desirable treat predicted numerous positive outcomes and achievements later in life, including interpersonal success, academic achievement, and SAT scores at a 12-year followup study [45]. Moffitt et al. [47] corroborated these findings in a larger sample of 1000 people, finding that self-control aptitude in childhood predicted a wide variety of outcomes in life domains such as health, finance, and criminality all the way up to the age of 32 . Notably, the cognitive capacity for self-control processes, which is closely linked to selfcontrol aptitude, plays an important role in self-regulation of behaviour [16,29]. Better cognitive control is linked to physical and mental well-being and the ability to successfully self-regulate outcomes such as academic and job achievements, addictions, and criminal and violent behaviour [14]. Given these findings, it seems plausible that even 
a small shift in a person's aptitude for self-control could have many important, long-term positive outcomes [47].

Games present a compelling opportunity for people to engage in tasks to measure and improve their capacity for self-control, yet researchers have not focused on how to incorporate various self-control tasks into games for these purposes. Despite the promising nature of games for selfregulation interventions, using such interactive designs has been limited to a small number of studies for improving the capacity for cognitive control without focusing on design aspects of a self-control game, or focusing on improving self-regulation of behaviour in everyday life [3]. As a first step toward designing a game to exercise and measure selfcontrol, we created a game to challenge various domains of self-control. We constructed a self-control activity in a game-like environment to investigate players' performance and experience in the game.

Constructing an activity that can effectively challenge selfcontrol processes presents a number of challenges. First, self-control tasks that require using cognitive control and effortful inhibition of responses are mentally taxing and possibly depleting $[5,19,27,48,50]$, which can negatively impact a player's experience. A game that requires considerable self-control might simultaneously create negative affect, decrease motivation, or result in disengagement from the task. We therefore conducted a study to explore our self-control game to demonstrate that (a) it can significantly target a player's cognitive capacity for self-control, and (b) did not affect other important factors of a positive player experience, such as disengagement or negative affect.

There are controversies surrounding the hypothesis that self-control can be improved simply by practicing a selfcontrol task $[32,33,40,41]$. In particular, it is highly debated whether the benefits of practicing one domain of selfcontrol transfers to other domains $[3,29,40,41]$ or helps to increase an overall limited resource [8-10,33,43]. For example, while working memory and inhibitory control are both considered related to self-control, it is uncertain whether exercising working memory has any effect on a different domain or whether it increases a limited resource that helps self-control generally. This poses a particular conundrum for the task of practicing self-control through a game, as at the core of that idea is the need to transfer this learned exercise outside of the game being played. While we do not presume to have a direct theoretical contribution to this highly debated topic, we suggest that games could be a suitable medium within which to exercise a variety of self-control processes simultaneously, and therefore remain a promising medium for self-control improvement. Thus, a second challenge is the design of self-control challenges that leverage multiple domains of self-control, rather than focusing on one domain.

In this work, we present the results of an initial user study, which showed that people exercise self-control in our game that challenges several self-control processes without reduc- ing their level of engagement, inducing negative affect, or undermining intrinsic motivation. This game essentially serves as a proof-of-concept that games can be designed to activate self-control processes without harming player experience. Our results also demonstrate that performance and player experience was connected to trait self-control, suggesting that games designed for self-control improvement or exercise could leverage this information to better target people with low vs. high levels of self-control.

\section{RELATED WORK}

We drew from self-control literature in psychology to design our initial study. Because this literature may be less familiar to readers, we review it in detail below. We then draw connections between our work and the current games literature and self-control improvement.

\section{Self-Control and Self-Control Processes}

In daily life, we all engage in the regulation of our thoughts, emotions, and behaviour to achieve desired states. The term self-regulation is often used more broadly to describe processes involved in goal-directed behaviour [29]. Selfcontrol has also been used interchangeably with selfregulation (e.g., [16]), but is often conceptualized as a subset of broader processes [29] for resolving goal-desire conflicts [22,23] or for altering and overriding our own responses "to bring them into line with standards such as ideals, values, morals, and social expectations, and to support the pursuit of long-term goals", [2, p. 351].

A broader level of top-down cognitive processes, known as executive functions (EFs) for cognitive control, arguably subsume the capacity for self-regulatory functions (for a review, see Hofmann et al. [29]). These self-regulatory abilities enable individuals to govern the self and pursue goaldirected purposes more generally [14]. The collection of these functions can be organized into three main categories: working memory, the holding and using of relevant information to our goals, and updating this in our mind; inhibitory control on responses over automatic and prepotent processes; and task switching, the mental flexibility to adapt to new circumstances and shift among a set of cognitive tasks to manage goals $[14,29]$. These processes play an important role as the capacity for self-regulation of behaviour [16,29] and are linked to physical and mental well-being and successfully self-regulating outcomes such as academic and job achievements, addictions, and criminal and violent behaviour (for a review, see Diamond [14]). In this paper, we also aim to target the cognitive capacity for successful selfregulation, similar to studies that intend to improve a person's cognitive capacity for self-control by practicing cognitive control tasks $[2,3,32,49]$.

A person who wishes to improve his or her capacity for self-regulation more broadly, encompassing all of the aforementioned self-regulatory functions, would still draw from more general self-regulatory strategies [15,20,51]. For example, in a self-control conflict, people need to evaluate choices and choose to resist an instant or short-term 
gratification of pursuing desires and tempting rewards or goals for a delayed reward, as in the Marshmallow Test [45]. It is important to note that the procedures of selfcontrol delay tasks such as the Marshmallow Test are different than of those in self-control executive function tasks that require cognitive control. However delaying gratification relies heavily on cognitive control and shares essential features [11,17]. For example, averting attention from tempting marshmallows and cookies involves inhibiting and overriding automatic and prepotent responses to control behaviours and thoughts [17]. It also involves the proper operation of working memory when encountering temptations [30]. These fundamental features indicate a link between successful self-regulation of behaviour and cognitive control [14,29].

The issue of self-regulation failure might look simple at first glance; however, a more in-depth understanding of psychological processes and strategies is required for understanding self-regulation, the causes of its failure, and mechanisms for improving it.

\section{Designing Games to Measure and Improve Self-Control}

Videogames are highly interactive media that can create long-term engagement and provide rich psychological experiences [53,56]. For these reasons, researchers have attempted to harness the power of videogames for a variety of purposes in altering human behaviour. Such attempts use game mechanics to encourage specific behaviours. For example, Foldit [12] is a game that encourages problem solving through crowdsourcing, encouraging players to invest significant effort at folding protein structures in the name of science. Systems have also been designed that use game elements, such as points, levels, and avatars to motivate behaviour. For example, HabitRPG [68] is a gamified system to improve productivity in managing tasks, habit, and other daily activities, and SuperBetter $[39,67]$ is designed to help people strengthen habits, tackle various personal or social life goals and challenges, and assist others.

To encourage such behaviours, researchers also have focused on influential theories of motivation, such as selfdetermination theory [55] and flow theory [13] which stress creating autonomous contexts, promoting player experience, and finding an optimal level of challenge. Other research, more in line with the objectives of this paper, has focused on improving mindfulness, which can facilitate affective well-being and self-regulation (e.g., [62]) through multiple mechanisms $[35,60]$. An example of this research in human-computer interaction research is PsychicVR, a design that uses brain-computer interfaces and virtual reality to provide real-time feedback, thus increasing mindfulness while creating a playful experience [1].

The work most closely related to ours has focused on improving self-control through interactive media either by testing the effects of existing commercial games or designing new games to improve self-control-that is, to improve specific cognitive control processes.
There are various categories of commercial games designed to improve numerous specific cognitive abilities, including cognitive control. On one hand, there are commercial games, known as "Brain Games," such as Big Brain Academy: Degree Wii, or games by Lumosity, promoted as a means of improving one's cognitive abilities. These games' appearance and media campaigns may lead people to think that they can actually improve one's cognitive abilities. However, a group of cognitive scientists recently issued a statement that there is little evidence to conclude "Brain Games" are effective and that they need further and broader investigation [69]. The same issue has led to a lawsuit against Lumosity creators and marketers [70]. Researchers have also attempted to improve attention and working memory by using action video games (e.g., Medal of Honor). While some studies have found no evidence to support the effectiveness of such games for the general public, others have found them to be effective for specific groups of people with impairments in cognitive control (for a review, see Anguera \& Gazzaley [3]). On the other hand, Neuroracer is a game designed for the purpose of improving cognitive control through multitasking and has been shown to improve specific aspects of cognitive control in older adults [2]. Prins et al. [52] also showed promising improvement in working memory in children with ADHD when using game elements to improve intrinsic motivation and self-efficacy (also, see Anguera \& Gazzaley [3]).

A number of studies that have attempted to investigate improvement in broader self-control processes have been based on the assumption that regularly practicing a simple task that requires self-control will improve overall selfcontrol capacity (e.g., practicing a handgrip task or using one's non-dominant hand for daily activities over the course of a few weeks; see [21,33] for review). This idea is based on the resource model of self-control [5] which conceptualizes that self-control relies on a limited pool of a resource and practicing self-control will increase this limited resource. However, these promising aspects of the resource model have recently been called into question by metaanalyses $[8,10]$ and failures to replicate a number of existing studies that initially provided evidence for its theoretical basis $[21,26,33,43]$. Thus, some research highlights the need for changing the approach of simply repeating a single task as an effective way to improve self-control [43]. Similarly, researchers have failed to find sufficient evidence that practicing working memory necessarily improves a person's overall capacity for cognitive control [40,42].

Lastly, we should note that the main concern of this branch of studies is improving a single specific domain of cognitive control, rather than improving behaviours that correspond to cognitive control more broadly. Therefore, there is a lack of research exploring whether games can impact realworld aspects of self-control and the self-regulated behaviours of everyday life by practicing self-control [36]. 

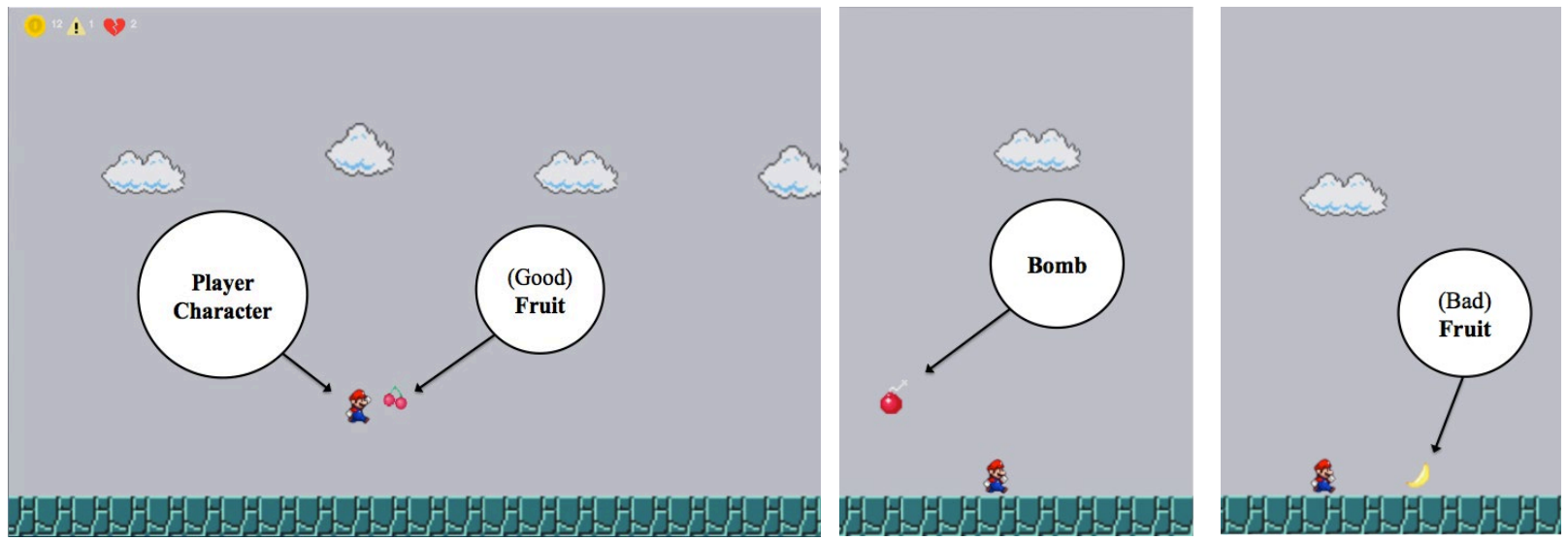

Figure 2. Screenshots of the game in go/no-go conditions of the study, including player character, (good and bad) fruits, and bomb items in the game. The player has jumped by pressing 'space' to collect a good fruit, has kept running to avoid a bomb, and needs to jump to avoid a bad fruit in this round of the game (from the left to the right snapshot respectively).

This research leads us to the following conclusions: First, a game that effectively improves self-control will need to present the right amount of challenge for a given player to ensure that their cognitive capacities and skills are being challenged and improved without decreasing engagement. Similarly, such a game would need to maintain quality of player experience to promote important motivational aspects in improving cognitive capacity. Further, such a game cannot rely simply on repeating a simple task in one domain of self-control if it is to improve cognitive control and a person's overall capacity for self-regulation of behaviour.

\section{SELF-CONTROL GAME DESIGN}

There are important advantages in using interactive technologies such as games for improving a person's selfcontrol capacity and executive function. Self-control processes are often effortful and possibly depleting [27,50], which makes it particularly difficult to maintain engagement, motivation, and positive affect when engaging in tasks requiring self-control. The motivational pull of videogames and their positive effect of momentary affective well-being $[53,56]$ make them a promising medium within which to embed interventions for self-control improvement. To explore the space of designing self-control games, we describe the design of our game, Save the Garden, and how we applied current theories of self-regulation and executive functions so that it targeted players' self-control.

\section{Game Design}

We used the Unity 4.5 game engine to design Save the Garden (Figure 2), a single-player platform game with two versions: one (go/no-go) was designed to engage different domains of self-control, and another ( $\mathrm{go}$ ) was designed so that it did not require self-control (and acted as a control condition in our study). We incorporated familiar game mechanics and characters into our design, and it resembles many commercial games, such as Subway Run, Flappy Bird, Circle, and Amazing Ninja in its mechanics.

\section{Go/No-Go Paradigm}

We based the design of our self-control game on the go/nogo paradigm [14], which is a commonly used paradigm in research on executive function (e.g., see Eigsti et al. [17]). A go/no-go task requires participants to respond to a stimulus by either choosing to act (go) or not act (no-go), and which is the "correct" choice alternates regularly. Because of this alternating pattern, the choice to not act requires withholding a response by inhibiting or overriding prepotent responses (e.g., in the presence of an automatic or dominant response $[29,46])$.

The main goal in the first game (go/no-go) is to collect as many "good" items as possible, and avoid collecting "bad" items. Therefore, players need to respond to items they want to collect (go events) and withhold a response by inhibiting or overriding prepotent responses to items they want to avoid (no-go events) [11,17]. In the second game (go), which acts as a control in our study, the main goal is to collect as many "good" items as possible, without any items to avoid.

\section{Save the Garden}

Each player starts game rounds in a garden with various items in it (e.g., fruits, small bombs, background items, etc.). In the go/no-go game, the character moves forward automatically, and game items appear on the screen as they move that either need to be collected or avoided by jumping using the space bar on the keyboard. Items can appear either at the same level or above the player, and so jumping or not jumping can alternately be required for collecting or avoiding. The type of item determines whether a person should collect or avoid an item (e.g., bombs should be avoided and good fruits should be collected). We use a familiar game character (Nintendo's Mario) to quickly communicate the narrative of the game.

A player in the go/no-go game collects all but one type of fruit (which has gone "bad" and must be avoided). There are six fruits in total, some similar in colour but not shape: 
apples, bananas, lemons, grapes, oranges, and cherries. The "bad" fruit changes every round and the player must also avoid bombs that have similar colours to some of the fruits in the game.

We designed the elements of the game in both conditions to be as similar as possible, including collectable items, character, scenario, and general goal. A player in the go game thus plays the same game, but has no "bad" fruits or bombs. However, in pilot tests with only this change, participants found the game too predictable and expressed a lack of engagement. We therefore made a few small adjustments to the go game to decrease boredom: fruits are behind or in front of semi-transparent trees (thus requiring some attention), characters can move back and forth (instead of automatic movement), and the fruits appear more regularly. We considered a variety of other mechanics, but finding a mechanic that is both engaging and has no systematic no-go response can be challenging. For example, platforms to jump on or across, action-at-a-distance mechanics (e.g., shooting guns/arrows), and other common platformer mechanics can require timing of when to "go" or "not go".

In addition to scores, the game provides auditory feedback. For instance, collecting good fruits results in a coincollecting sound, collecting bad fruits results in an error sound, and collecting a bomb results in a small flash effect in the screen with an "ouch" sound. These sounds are informational and help players to learn how to play.

\section{Use of Self-Control in Save the Garden}

While we base our design on the go/no-go paradigm, our game goes beyond using a single EF task by incorporating more complex mechanics in a realistic game-like setting. Therefore, players require multiple interrelated components of self-control to perform well in the game. They need to use working memory to maintain, use, and update information about multiple unfamiliar fruits in the game to collect or avoid them. There is an additional task switching cost when the player starts a new round and the "bad" fruits change, which means the correct response in the first round could be an incorrect response in the following rounds, and vice versa. Players need to use inhibitory control to inhibit various responses to avoid bombs and "bad" fruits. We make this inhibition more difficult by using similar colours for bombs and fruits. Furthermore, the ratio of randomized fruits varies in the game. Thus, players are required to use a broad range of self-control processes to perform well. It is also likely that players need to regulate their interfering thoughts or negative emotions (e.g., in-between rounds when they perform poorly in a round), especially when they compare this performance to previous rounds.

\section{Generalizability of Save the Garden}

One challenge in designing a game that incorporates selfcontrol mechanics is to not lose the essence of what makes it a "game". Goals and objectives, rules, and decisionmaking that result in quantifiable and clear outcomes are considered to be central in many definitions of a game [57], which our self-control game satisfies. Nonetheless, incorporating these mechanics into something more complex, like Skyrim or the Civilization series would require significant effort. However, our proof-of-concept shows that games with simpler mechanics and lower fidelity (e.g., Subway Run, Super Mario Run, Temple Run) can already incorporate self-control mechanics, and future research could explore more complex, higher-fidelity games.

\section{STUDY OF SELF-CONTROL GAMES}

We wanted to investigate whether we were successful at designing a game that can challenge self-control, without negatively impacting other important elements of an effective self-control game, such as engagement, negative affect, or intrinsic motivation. In addition to these primary goals, we examined the effect of player performance on each of these constructs. We also investigated individual differences in players' self-reported chronic levels of self-control to see whether these differences were related to performance in the game.

Our primary research question focused on designing games intended to challenge a range of self-control processes:

RQ1. Do participants exercise self-control when playing the self-control (go/no-go) game?

We were also interested in whether challenging self-control processes might undermine player experience. We therefore also asked:

RQ2. Does playing the self-control game undermine engagement and quality of player experience?

Furthermore, we wanted to explore how designers might take into account a player's trait self-control. We therefore investigated the connection between trait self-control and both measures of performance that could be used to adapt the game, such as rates of error, and possible effects adjustments might have on player experience. We thus asked:

RQ3. What is the effect of trait self-control on performance and quality of experience?

RQ4. How does performance affect the quality of player experience in self-control games?

\section{Method}

We conducted a lab study to examine these research questions, and therefore whether self-control games have the potential to be successful.

\section{Participants}

We recruited 45 university students (19 female; 25 male; 1 not specified) to participate in a study of "Game Playing Experience" through campus flyers. They were randomly assigned to two conditions (22 go/no-go condition; 23 go condition). All participants completed the study and were paid $\$ 10$ for their time. 


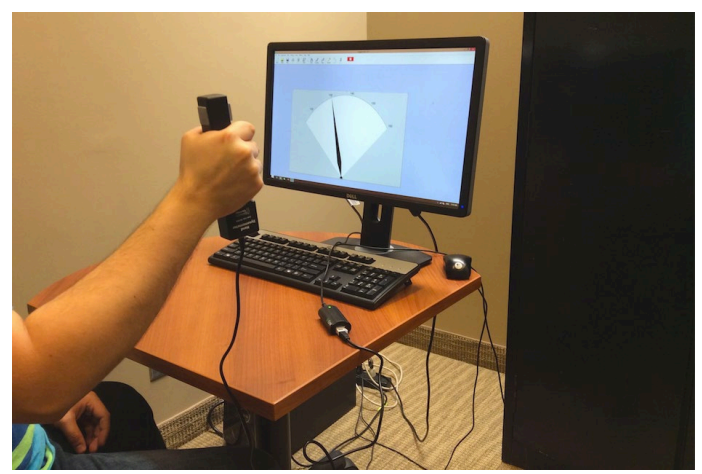

Figure 3. The digital hand dynamometer used to measure handgrip performance.

\section{Procedure}

We used a dual-task paradigm [50] that examines the effect of one task in which people exert self-control on an unrelated subsequent task that also requires self-control. This approach uses a between-groups design with half the participants in a self-control condition (go/no-go) and the other half in a control condition ( $g o$ ). The expectation in this paradigm is that exerting self-control in one task will result in poorer performance in the subsequent task [50].

This effect has been theoretically attributed to different causes, such as depletion of a limited capacity for selfcontrol [5,50], a shift in motivational priorities and attention [34], or an opportunity-cost of an action [37]. Our primary objective, however, was not to investigate the underlying theory, but to provide initial confirmation that our manipulation is strong and effective enough to engage participants in a complex self-control task. Therefore, please note that we do not suggest that using this paradigm is necessary for future HCI researchers that design self-control games.

We used a handgrip task as the subsequent task (using a digital hand dynamometer; Figure 3), which has been frequently used in dual-task paradigms [19,27,48,50]. The ability to hold a handgrip for a prolonged period of time is related to persistence rather than simple physical strength because the task requires endurance as an individual resists the urge to quit due to physical discomfort [54,64], although strength must be controlled for statistically. In our study, we used a threshold of $2 / 3$ of a their maximum strength to account for variance in physical strength [64].

The sequence for each participant was as follows (Figure 4): they first completed a handgrip task; then, after a short training session to familiarize themselves with the basic movements and features and the goal of the game, each participant played six rounds of one of the two game versions (about 15 minutes); then, there was an interim period to separate the two tasks $[27,50]$ for about 2.5 minutes, in which we had participants complete the PANAS questionnaire that measures post-game affect before completing the second handgrip task. We collect data about affect in this interim period to better control for the effect of gameplay on the subsequent task in the dual-task paradigm, for exam-

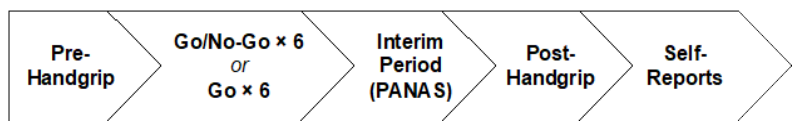

Figure 4. The experimental procedure.

ple, if the negative affect from playing a difficult game were to affect handgrip performance [50]. Finally, each participant completed a longer set of questionnaires. Participants were not specifically instructed to compete with other participants in different sessions of the experiment nor they were aware of others' performance; however, being a game environment, participants were observed to be motivated to perform well in the game.

\section{Self-Report Measures}

After playing the game, participants completed the Positive and Negative Affect Schedule (PANAS; [65]). We also asked participants about their level of engagement in the game by using the immersion item, "I really got into the game", from the Game Engagement Questionnaire (GEQ; [7]). We also asked about how challenging and difficult participants found the game to be. Finally, participants completed a version of the Intrinsic Motivation Inventory (IMI) to mainly investigate interest/enjoyment, perceived competence, tension/pressure subscales. This scale was validated by McAuley et al. [38] and serves to examine the quality of player experience. We calculated Cronbach's Alpha for each of the IMI subscales; all reliability scores were acceptable ( $\alpha>$.84, for all four subscales).

Participants were also asked two questions about their perceived experience in playing games in general and their average number of hours playing videogames per week. Participants were then asked to complete the 36-item SelfControl Scale (SCS) [63], a widely used scale to measure individual differences in trait self-control $(\alpha=.80)$. They also completed a Morningness-Eveningness Scale [31], which is used to measure individuals' circadian rhythms, to control for any impact this may have had on their performance at different times of the day.

\section{Game Performance}

We logged the number of errors that a player made in each round of the game. The types of errors included: missing a good fruit (generally referred to as an omission error), collecting a bad fruit (a commission error), or hitting a bomb (another commission error). The appearance and order of items were pseudo-randomized in the game, and the total number of items in each session was constant. The go/no-go condition had all three types of errors, but the go condition only had omission errors (missing good fruits). In both conditions, we used the total error (error-total) as a measure of performance in the game.

\section{RESULTS}

We first present the results of our primary dual handgrip task, followed by an analysis of the self-report measures. We then present an analysis of the performance data, first for the go/no-go condition, then for the go condition. In our 


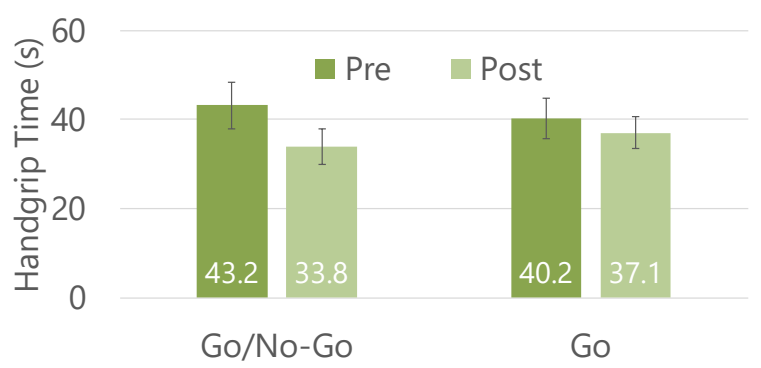

Figure 5. Pre- and post-condition handgrip times.

analysis of performance data, we included correlational and mediation analyses that incorporated self-report measures. The order of this analysis is presented in roughly the same order as our research questions (RQ1-RQ4).

\section{Handgrip}

RQ1. Do participants exercise self-control when playing the self-control (go/no-go) game? We calculated the difference between pre- and post-condition handgrip time $(\Delta \mathrm{t})$ as a measure to calculate differences in handgrip performance, and compared the two groups using a $t$-test. There was a significant difference between the go/no-go and go conditions, $t_{36}=2.14, p=.039$ (go/no-go: $M=-10.7 \mathrm{~s}, S D=$ $14.1 \mathrm{~s}$, go: $M=-0.7 \mathrm{~s}, S D=16.4 \mathrm{~s}$; Cohen's $d=.65)$, indicating that the participants in the go/no-go condition performed worse than those in the go condition as indicated by a larger decline in pre- to post-handgrip performance. We ran a 2 (game type: go, go/no-go) $\times 2$ (measurement time: pre, post) mixed model RM-ANOVA on the handgrip time measure that confirmed the same significant interaction between game type and measurement time $\left(F_{1,36}=4.59\right.$, $\left.p=.039, \eta_{p}^{2}=.116\right)$, which remained robust even when controlling for negative affect $\left(F_{1,36}=4.14, p=.049, \eta_{p}^{2}=.106\right)$, positive affect $\left(F_{1,36}=4.65, p=.038, \eta_{p}^{2}=.117\right)$, or Morningness-Eveningness $\left(F_{1,36}=5.80, p=.021, \eta_{p}^{2}=.142\right)$ as covariates in the analysis. This result shows that playing the go/no-go version of the game resulted in worse performance post-game in a secondary handgrip self-control task than the go version.

Six participants did not accurately follow the handgrip performance procedure in either the pre- or post-condition handgrip tests, and one participant was unable to perform the handgrip task for health reasons, and so were excluded from the analysis ${ }^{1}$.

\footnotetext{
1 The only individual differences between excluded participants and others, interestingly, were that they played higher hours of weekly video game playing, $F_{1,44}=$ $16.270, p<.001, \mathrm{~d}=1.12$. They also perceived themselves as more experienced in playing video games, $F_{1,44}=4.590, p=.021, \mathrm{~d}=1.25$. Perhaps, this suggests that they might have been mostly just interested in playing a game in an experiment, therefore paid less attention to the non-game physical tasks of the experiment. Notably, the rate of exclusion in the current study $(\approx 15 \%)$ was not high when compared to some other dual-task paradigms investigating depletion (e.g., an average of $26 \%$ exclusion across 23 labs [27]; also see [24]).
}

\section{Self-Report Measures}

RQ2. Does playing the self-control game undermine engagement and quality of player experience? The results of our analysis of player experience in the game are illustrated in Figure 6. We ran a multivariate analysis of variance (MANOVA) on the subscales of the self-report measures of player experience as the eight dependent variables with game type as a fixed factor. We subsequently ran protected independent-samples $t$-tests to compare responses between the go/no-go and go groups (using Bonferroni corrected $\alpha=$ $.05 / 8=.00625)$. As expected, participants in go/no-go perceived the game to be significantly more challenging, $t_{43}=$ $3.12, p=.003, d=1.93$. However, the perceived level of challenge in the go/no-go condition was still approximately at the mid-low level $\left(M_{\text {go/no-go }}=3.6, S D_{\mathrm{go} / \mathrm{no}-\mathrm{go}}=1.5 ; M_{\mathrm{go}}=\right.$ 2.3, $S D_{\text {go }}=1.4$, Figure 6a). There was no significant difference in the post-game reported level of engagement (immersion) between the two groups, $t_{43}=0.09, p=.77, n s$, and both groups indicated mid-level engagement with the game (Figure 6a).

We found no significant difference between the groups in post-game reported interest/enjoyment, $t_{43}=-1.56, p=.13$ $\left(M_{\mathrm{go} / \mathrm{no}-\mathrm{go}}=3.6, S D_{\mathrm{go} / \mathrm{no}-\mathrm{go}}=1.2 ; M_{\mathrm{go}}=4.2, S D_{\mathrm{go}}=1.2\right.$, Figure $6 b)$. Participants in the go condition, as expected, had higher perceived competence about their performance in the game than the go/no-go group $t_{43}=-3.88, p<.001$. However, both groups indicated at least a medium level of competence, $M_{\mathrm{go} / \mathrm{no}-\mathrm{go}}=4.2, S D_{\mathrm{go} / \mathrm{no}-\mathrm{go}}=1.2 ; M_{\mathrm{go}}=5.4, S D_{\mathrm{go}}=1.0$. Notably, participants in the go/no-go condition reported experiencing higher tension and pressure when playing the game, as shown in Figure $6 \mathrm{~b}, t_{43}=2.98, p=.005, d=.88$, even though their pressure/tension levels were not high $\left(M_{\mathrm{go} / \mathrm{no}-\mathrm{go}}=4.1, S D_{\mathrm{go} / \mathrm{no}-\mathrm{go}}=1.6 ; M_{\mathrm{go}}=2.8, S D_{\mathrm{go}}=1.2\right)$. Finally, there was no significant difference in post-game negative affect, $t_{43}=0.71, p=.49, n s .\left(M_{\mathrm{go} / \mathrm{no}-\mathrm{go}}=1.46, S D_{\mathrm{go} / \mathrm{no}-\mathrm{go}}\right.$ $\left.=.38 ; M_{\mathrm{go}}=1.39, S D_{\mathrm{go}}=.35\right)$, , or positive affect, $t_{43}=-.243$, $p=.81, n s$. We did not analyze individual differences in Morningness-Eveningness on player experience and performance; this is planned for future work. Notably, this result shows that self-reports on engagement, enjoyment, and positive or negative affect were not statistically different between go and go/no-go, despite participants exerting self-control in the go/no-go game.

\section{Go/No-Go Performance \& Correlations}

In the go/no-go group, the proportion of errors was calculated as the sum of three types of errors across all the rounds (error-total) divided by the total number of items. $(M=9.4 \%, S D=4.2 \%$,Figure 7$)$, which showed an acceptable internal reliability $(\alpha=.82)$. The go condition had only one type of error, and so we analyzed data from both groups separately. We conducted planned pairwise comparisons of sequential rounds ( 5 total comparisons) to explore player performance, which revealed significant differences between rounds 1 and $2, t_{21}=2.9, p<.01$, and rounds 2 and $3, t_{21}=2.4, p=.02$. This pattern is consistent with a learn- 


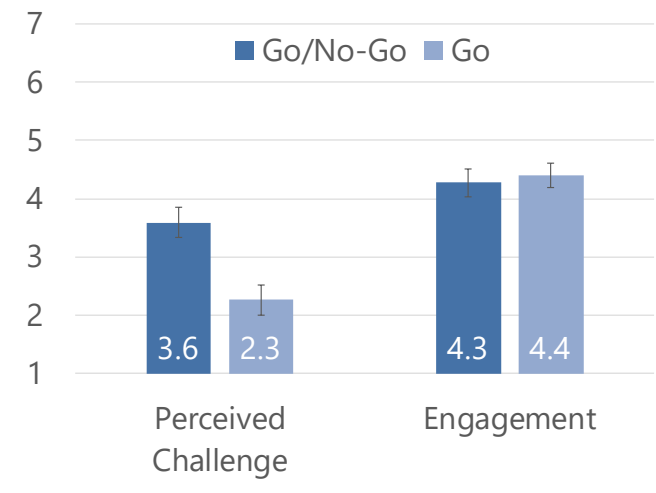

(a) Perceived Challenge/Engagement

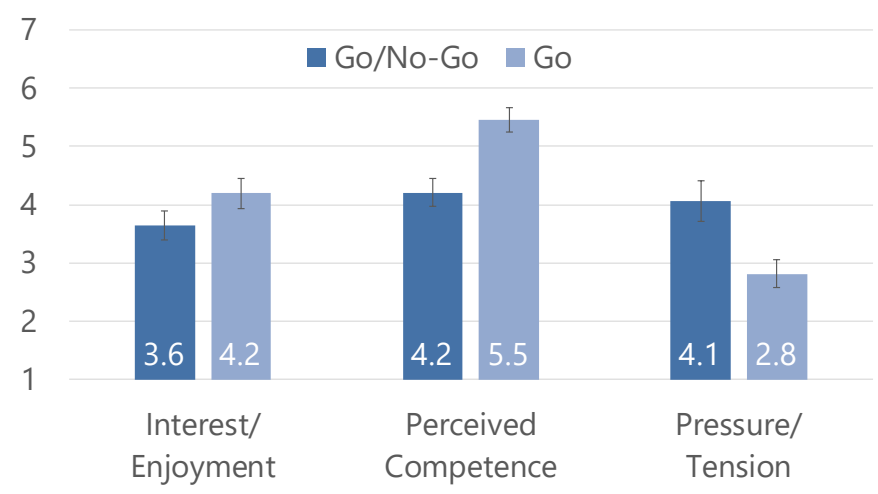

(b) Intrinsic Motivation Inventory

Figure 6. Player experience and Intrinsic Motivation Inventory results on both conditions. Error bars represent \pm 1 standard error of the mean.

ing effect where performance improves until round 3 and then remains consistent (Figure 7).

To investigate the relationship of performance with player experience and trait-level of self-control (RQ2-RQ3), we conducted correlational and mediation analyses.

\section{Correlational Analysis}

$R Q 3$. What is the effect of trait self-control on performance and quality of experience? Pearson correlations indicate that participants with higher levels of trait self-control had higher levels of perceived competence $(\mathrm{N}=22, \mathrm{r}=.466$, $\mathrm{p}=.029)$. Notably, trait self-control was also marginally correlated $(\mathrm{p}<.06)$ with fewer errors $(\mathrm{N}=22, \mathrm{r}=-.413$, $\mathrm{p}=.056)$, and lower perceived challenge in the game $(\mathrm{N}=22, \mathrm{r}=-.418, \mathrm{p}=.053)$. The total number of errors in the game was not correlated with perceived experience in video game playing $(\mathrm{N}=22, \mathrm{r}=-.111, \mathrm{p}=.623)$ or number of hours played per week $(\mathrm{N}=22, \mathrm{r}=.093, \mathrm{p}=.679)$. This result shows that trait self-control predicted game performance and perceived challenge for the go/no-go game.

RQ4. How does performance affect the quality of player experience in self-control games? Players with fewer total errors had significantly higher perceived competence

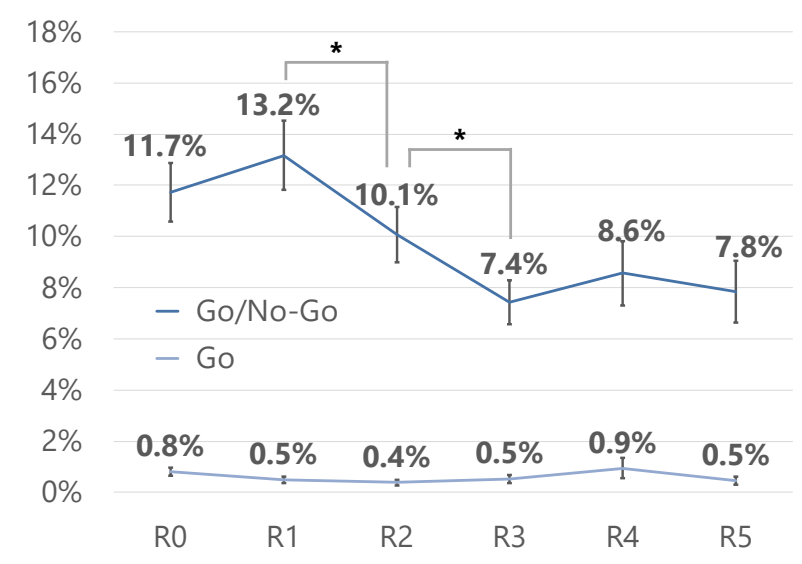

Figure 7. Performance in the go/no-go and go conditions. Participants performed worse in early rounds, and improved in later rounds, but only in go/no-go. * $p<.05$
$(N=22, r=-.588, p=.004)$ as expected. Players with fewer errors also had significantly higher levels of interest/enjoyment in the game $(N=22, r=-.487, p=.021)$, but did not experience a significant difference in pressure or tension $(N=22, r=-.281, p=.205)$. This indicates that game performance impacted the level of perceived competence and enjoyment in the go/no-go game.

To investigate how each error type in the game might have influenced the level of perceived competence and enjoyment, we also analysed the relationship between omission errors (type 1: missing a good fruit), and commission errors (type-2: collecting a bad fruit, type-3: collecting a bomb) with IMI measures. Results show that all error types contributed to the level of perceived competence in the game. Perceived competence was significantly correlated with number of missed good fruits $(N=22, r=-.427, p=.047)$, collected bad fruits $(N=22, r=-.562, p=.006)$, and marginally with the number of collected bombs $(N=22, r=$ $.418, p=.053)$. Also, the level of interest/enjoyment in the game was marginally correlated with all error types: number of missed good fruits $(N=22, r=-.410, p=.058)$, collected bad fruits $(N=22, r=-.379, p=.082)$, and collected bombs $(N=22, r=-.382, p=.079)$.

\section{Mediation Analysis}

We further investigated the relationship between trait selfcontrol, players' game performance (error-total), and their perceived competence in the game using a mediation analysis (Figure 8). The level of trait self-control can directly predict higher perceived competence, $\beta=1.10,(C I=.13$, $2.07), t_{21}=2.4, p=.03$. As previously mentioned, trait selfcontrol predicts the total number of errors (performance), $\beta$ $=-2.43,(C I=-4.93, .07), t_{21}=-2.0, p=.056$ which itself is a significant predictor of perceived competence, $\beta=-.236$, $(C I=-.39,-.09), t_{21}=-3.3, p=.004$. We used hierarchical regression analysis to examine the effects of trait selfcontrol when controlling for game performance. The direct prediction of trait self-control does not stay significant, $\beta=$ $.63,(C I=-.32,1.59), t_{21}=1.39, p=.18$. A clear limitation of this analysis is the sample size; however, we decided to include this analysis as an initial investigation of this im- 


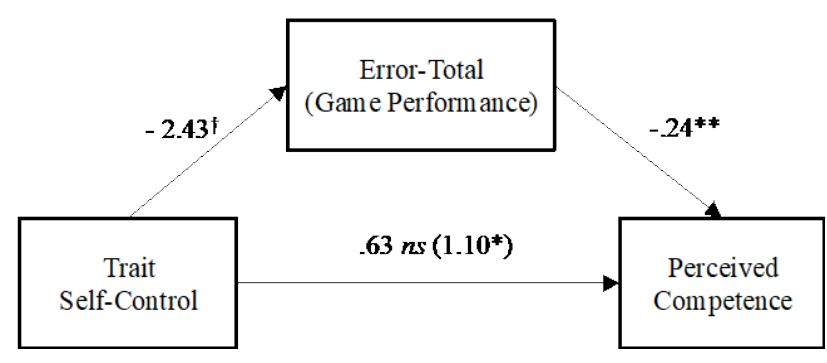

Figure 8. The mediation analysis of the relationship between trait self-control, perceived competence, and game performance. Numbers represent unstandardized beta values. $N=\mathbf{2 2}$, ${ }^{\dagger} p<.06,{ }^{*} p<.05, * * p<.01$

portant relationship. This result shows that game performance (error-total) mediates the effect of trait self-control on perceived competence in this game. The result of a biascorrected accelerated bootstrap, that adjusts bias or skewness in its distribution, also confirms the same level of significance reported in Figure 8.

\section{Go Performance \& Correlations}

Although the go condition requires some degree of attention, players had a low rate of error $(M=0.45 \%, S D=$ $0.80 \%$ ), and there was no significant difference between the rounds $\left(t_{21}<1.74, p>.09\right)$. Interestingly, players in the $g o$ condition who had higher trait self-control experienced more positive affect after playing $(N=23, r=.414$, $p=.050)$. In general, participants in the go/no-go condition found the game more challenging than those in the go condition (see Self-Report Measures). However, in the go condition, the reported level of engagement is marginally correlated with how challenging they found the game $(N=23$, $r=.407, p=.054)$.

\section{DISCUSSION}

Important findings of our study are summarized as follows:

- Playing the go/no-go version of our game resulted in worse performance post-game in a secondary handgrip self-control task than the go version.

- Despite exerting self-control in the go/no-go game, self-reports on engagement, enjoyment, and positive or negative affect were not statistically different between $g o$ and go/no-go.

- Participants reported higher perceived challenge and higher pressure/tension in the go/no-go condition.

- Trait self-control predicted game performance and perceived challenge for the go/no-go game.

- Game performance impacted the level of perceived competence and enjoyment in the go/no-go game.

- Participants had a pattern of play in the go/no-go game with improved performance in the self-control task we provided, but similar differences between rounds were not observed in the go group.

- Trait self-control predicted perceived competence, but was mediated by game performance for go/no-go.
The results of the study are promising and show the possibility of designing self-control games that engage a broad set of self-control processes, without negatively affecting player experience or causing disengagement. In particular, our game allowed people to exercise self-control by engaging them in multiple domains of self-control processes in a themed self-control exercise task within a realistic gamelike setting. Differences in handgrip performance show that the go/no-go group exercised effortful self-control.

Our results showed a similar rate of error as other research with simpler go/no-go paradigms (e.g., [17]). However, our work adds to these results by demonstrating that players reported an acceptable level of engagement with the game that remained consistent as time progressed, and performance did not drop in later trials, for example, as the result of boredom. Notably, the level of engagement was not significantly correlated with any other factor, including level of trait self-control or number of errors in the game that required self-control. This result shows that it may be possible to engage players with different levels of self-control, which paves the way for further investigation into the design of games that target self-control, but also allow for long-term engagement.

Our results also indicate that the level of self-control of players in the go/no-go condition can predict performance in the game. This finding is corroborated by the result that players with higher trait self-control perceived the game as less challenging. These results have two important implications: 1) They provide insight on the possibility of designing for improvement in general domains of self-control, even using an activity that does not use particularly difficult challenges; 2) It shows initial evidence into the possibility of measuring trait-level self-control that could be used in an adaptive design to meaningfully and dynamically adjust the challenges with respect to both the level of self-control and progress in the game. For example, players that are performing well are likely to be high in trait self-control, and the game can adjust to present more challenging selfcontrol tasks (e.g., as in [66]). This technique may be valuable when designing games to improve a person's selfcontrol capacity and executive functions, and usually cannot be achieved by more traditional cognitive training tasks (e.g., $[6,16])$. The purpose of these results is not to introduce a new methodology to measure self-control in games, but to encourage future researchers to consider this as a strong possibility for designing self-control games.

Moreover, our results suggest a connection between specific measures of performance (omission and commission errors) and both perceived competence and level of enjoyment. Thus, this dynamic adjustment may also better target players' basic needs, and our game demonstrates low-level performance measures that could be used in this manner.

One risk of designing a game that targets self-control is that people with lower levels of self-control can potentially experience lower perceived competence. For instance, as they 
are less capable of regulating their negative thoughts or moods [58], they may require special mechanisms (e.g., more positive feedback) to mitigate and increase perceived competence. However, our mediation analysis showed that the number of errors (game performance) mediated the effect of self-control on perceived competence in the game, which indicates that players have a fairly accurate perception of their competence in the game based on the information they receive. Therefore, promisingly, it may be possible for a designer to not require special in-game mechanics to address players with low trait self-control.

\section{LIMITATIONS AND FUTURE WORK}

Our work relies on an underlying theory of self-control that involves the development of several self-control processes. While improvement of any one of these processes has the potential for numerous positive effects, especially if a person has known weaknesses in that process $[3,29]$, it is important to note that, although people with higher cognitive capacity for self-regulation are better at self-regulation of their behaviour [14,29], this might not reflect all aspects that lead to success in self-regulation. In other words, although effortful inhibition of responses to temptations might be a necessary condition and an essential mediator to succeed, it might not be a sufficient condition nor the most effective way to self-regulate behaviour $[22,44]$.

On the other hand, recent studies point out that people with higher trait self-control can strategically avoid temptation and experience less temptation in the long-run, as they select and evaluate situations they face in life better than others $[15,18,44]$. Thus, there is some evidence that practicing a variety of self-control processes in a game-like environment has the potential to improve self-regulatory processes more broadly, and thus lead to success in other parts of life.

There are many promising avenues of future work in the endeavour to create game-like environments for self-control improvement. In particular, games have the potential to create real-life situations and scenarios, where players can adopt different short-term and long-term goals. Designing games in which players experience real choices and choice dilemmas have the potential to lead to meta-cognitive improvements in life. Future work could explore how integrating these more realistic dilemmas with self-control challenges might better simultaneously target improvements that lead to successful self-regulation.

\section{GENERAL DISCUSSION}

Our paper has mostly focused on positive aspects of targeting people's self-control for the purpose of self-control improvement. However, there might be short-term costs to playing such games. For instance, incorporating self-control elements into games might have an effect on problematic gaming behaviour. Self-regulation failure is associated with various aspects of problematic online behaviour $[25,59]$ that could negatively impact players both during and after play. Therefore, practicing self-control might have an impact on some short-term behaviours and decisions, especially in- game, for example, making unwanted in-app-purchases as the result self-regulation failure [61]. It is therefore important to investigate other effects of using self-control game elements on players' gaming experience and behaviour, for example, how this knowledge could be exploited by game designers to indulge players into spending more money while playing games.

However, even though self-control practice over a period of gameplay might cause cognitive depletion or a shift in attentional and motivational processes and therefore affect subsequent activities or decisions [5,34,50], these effects are not expected to carry over to a longer period of time (e.g., the whole day). That is, practicing self-control does not necessarily result in a long period of cognitive fatigue [33]. A useful metaphor of the strength model of selfcontrol is its resemblance to a muscle [5]. It can be thought of as improving muscle strength at the short-term cost of using muscles - the costs of practicing self-control games can be thought of as the short-term costs of exercising that may lead to long-term benefits.

\section{CONCLUSION}

Videogames have the potential to integrate elements for successfully improving and measuring self-control. This provides numerous possibilities to design self-control games beyond existing approaches. Our work has made a number of contributions to the design and evaluation of self-control games. In particular, we provide initial empirical evidence that it is possible to engage players in selfcontrol games without creating a negative player experience or undermining intrinsic motivation. Our work is one of the first steps in the investigation of self-control games-games that aim to improve self-control-which is an endeavour that has the potential to improve people's ability to pursue their goals and increase overall well-being.

\section{ACKNOWLELDGEMENTS}

We thank the Natural Sciences and Engineering Research Council of Canada (NSERC), Saskatchewan-Waterloo Games User Research (SWaGUR) program, Social Sciences and Humanities Research Council of Canada (SSHRC), and Ontario Ministry of Research and Innovation for funding.

\section{REFERENCES}

1. Judith Amores, Xavier Benavides, and Pattie Maes. 2016. PsychicVR. In Proceedings of the 2016 CHI Conference Extended Abstracts on Human Factors in Computing Systems - CHI EA '16 (2016). http://dx.doi.org/10.1145/2851581.2889442

2. Joaquin A. Anguera et al. 2013. Video game training enhances cognitive control in older adults. Nature 501, 7465 (April 2013), 97-101. http://dx.doi.org/10.1038/nature12486

3. Joaquin A. Anguera and Adam Gazzaley. 2015. Video games, cognitive exercises, and the enhancement of cognitive abilities. Current Opinion in Behavioral 
Sciences 4 (2015), 160-165.

http://dx.doi.org/10.1016/j.cobeha.2015.06.002

4. Roy F. Baumeister, Todd F. Heatherton, and Dianne M. Tice. 1994. Losing control: How \& why people fail at self-regulation and Handbook of self-regulation. Academic press. https://doi.org/10.1016/0272-7358(95)90149-3

5. Roy F. Baumeister, Kathleen D. Vohs, and Dianne M. Tice. 2007. The strength model of self-control. Current Directions in Psychological Science 16, 6 (2007), 351355.

http://dx.doi.org/10.1111/j.1467-8721.2007.00534.x

6. Blair Saunders, Marina Milyavskaya, Alexander Etz, Daniel Randles, and Michael Inzlicht. 2018. Reported self-control does not meaningfully assess the ability to override impulses.

https://doi.org/10.17605/OSF.IO/BXFSU

7. Jeanne H. Brockmyer, Christine M. Fox, Kathleen A. Curtiss, Evan Mcbroom, Kimberly M. Burkhart, and Jacquelyn N. Pidruzny. 2009. The development of the Game Engagement Questionnaire: A measure of engagement in video game-playing. Journal of Experimental Social Psychology 45, 4 (2009), 624634. http://dx.doi.org/10.1016/j.jesp.2009.02.016

8. Evan C. Carter and Michael E. Mccullough. 2014. Publication bias and the limited strength model of selfcontrol: has the evidence for ego depletion been overestimated? Frontiers in Psychology. 5 (2014). http://dx.doi.org/10.3389/fpsyg.2014.00823

9. Evan C. Carter and Michael E. Mccullough. 2013. Is ego depletion too incredible? Evidence for the overestimation of the depletion effect. Behavioral and Brain Sciences 36, 06 (2013), 683-684. http://dx.doi.org/10.1017/s0140525x13000952

10. Evan C. Carter, Lilly M. Kofler, Daniel E. Forster, and Michael E. Mccullough. 2015. A series of metaanalytic tests of the depletion effect: Self-control does not seem to rely on a limited resource. Journal of Experimental Psychology: General 144, 4 (2015), 796 815. http://dx.doi.org/10.1037/xge0000083

11. B.J. Casey et al. 2011. Behavioral and neural correlates of delay of gratification 40 years later. Proceedings of the National Academy of Sciences 108, 36 (2011), 14998-15003. http://dx.doi.org/10.1073/pnas.1108561108

12. Seth Cooper et al. 2010. Predicting protein structures with a multiplayer online game. Nature 466, 7307 (May 2010), 756-760.

http://dx.doi.org/10.1038/nature09304

13. Mihaly Csikszentmihalyi and Judith Lefevre. 1989. Optimal experience in work and leisure. Journal of Personality and Social Psychology 56, 5 (1989), 815822. http://dx.doi.org/10.1037//0022-3514.56.5.815
14. Adele Diamond. 2013. Executive functions. Annual Review of Psychology. 64, 1 (March 2013), 135-168. http://dx.doi.org/10.1146/annurev-psych-113011143750

15. Angela L. Duckworth, Tamar S. Gendler, and James J. Gross. 2016. Situational strategies for self-control. Perspectives on Psychological Science 11, 1 (January 2016), 35-55.

http://dx.doi.org/10.1177/1745691615623247

16. Angela L. Duckworth and Margaret L. Kern. 2011. A meta-analysis of the convergent validity of self-control measures. Journal of Research in Personality 45, 3 (2011), 259-268. http://dx.doi.org/10.1016/j.jrp.2011.02.004

17. Inge-Marie Eigsti et al. 2006. Predicting cognitive control from preschool to late adolescence and young adulthood. Psychological Science 17, 6 (January 2006), 478-484.

http://dx.doi.org/10.1111/j.1467-9280.2006.01732.x

18. Michael R. Ent, Roy F. Baumeister, and Dianne M. Tice. 2015. Trait self-control and the avoidance of temptation. Personality and Individual Differences 74 (2015), 12-15.

http://dx.doi.org/10.1016/j.paid.2014.09.031

19. Eli J. Finkel, W. Keith Campbell, Amy B. Brunell, Amy N. Dalton, Sarah J. Scarbeck, Tanya L. Chartrand, and Sarah J Scarbech. 2006. Highmaintenance interaction: Inefficient social coordination impairs self-regulation. Journal of Personality and Social Psychology 91, 3: 456-475. https://doi.org/10.1037/0022-3514.91.3.456

20. Ayelet Fishbach and Benjamin A. Converse. 2010. Identifying and battling temptation. In Handbook of self-regulation: Research, theory and applications (2nd ed.). 244-260.

21. Malte Friese, Veronika Job, Julius Frankenbach, and David D. Loschelder. Meta-analysis of self-control training. 2016. (Unpublished Manuscript)

22. Kentaro Fujita. 2011. On Conceptualizing Self-Control as More Than the Effortful Inhibition of Impulses. Personality and Social Psychology Review 15, 4: 352366.

https://doi.org/10.1177/1088868311411165

23. Kentaro Fujita, Yaacov Trope, Nira Liberman, and Maya Levin-Sagi. 2006. Construal levels and selfcontrol. Journal of personality and social psychology 90, 3: 351-67.

https://doi.org/10.1037/0022-3514.90.3.351

24. Chandra Sripada, Daniel Kessler, and John Jonides. 2016. Sifting Signal From Noise With Replication Science. Perspectives on Psychological Science 11, 4 (2016), 576-578. http://dx.doi.org/10.1177/1745691616652875 
25. Maria C. Haagsma, Scott E. Caplan, Oscar Peters, and Marcel E. Pieterse. 2013. A cognitive-behavioral model of problematic online gaming in adolescents aged 12-22 years. Computers in Human Behavior. https://doi.org/10.1016/j.chb.2012.08.006

26. Martin .S. Hagger et al. 2016. A multi-lab preregistered replication of the ego-depletion effect. Perspectives on Psychological Science 11, 4 (January 2016), 546-573. http://dx.doi.org/10.1177/1745691616652873

27. Martin S. Hagger, Chantelle Wood, Chris Stiff, and Nikos L.D. Chatzisarantis. 2010. Ego depletion and the strength model of self-control: A metaanalysis. Psychological Bulletin 136, 4 (2010), 495 525. http://dx.doi.org/10.1037/a0019486

28. Wilhelm Hofmann, Maike Luhmann, Rachel R. Fisher, Kathleen D. Vohs, and Roy F. Baumeister. 2013. Yes, but are they happy? Effects of trait self-control on affective well-being and life satisfaction. Journal of Personality 82, 4 (August 2013), 265-277. http://dx.doi.org/10.1111/jopy.12050

29. Wilhelm Hofmann, Brandon J. Schmeichel, and Alan D. Baddeley. 2012. Executive functions and selfregulation. Trends in Cognitive Sciences 16, 3 (2012), 174-180. http://dx.doi.org/10.1016/j.tics.2012.01.006

30. Wilhelm Hofmann, Brandon J. Schmeichel, and Alan D. Baddeley. 2011. Working Memory and SelfRegulation. In Handbook of self-regulation: Research, theory and applications (2nd ed.). 204-225.

31. Jim A. Horne and Olov Östberg. 1975. A selfassessment questionnaire to determine morningnesseveningness in human circadian rhythms. International journal of chronobiology, 4, 2 (1975), 97-110.

32. Michael Inzlicht, Lisa Legault, and Rimma Teper. 2014. Exploring the Mechanisms of Self-Control Improvement. Current Directions in Psychological Science 23, 4 (2014), 302-307. http://dx.doi.org/10.1177/0963721414534256

33. Michael Inzlicht and Elliot Berkman. 2015. Six questions for the resource model of control (and some answers). Social and Personality Psychology Compass 9, 10 (2015), 511-524. http://dx.doi.org/10.1111/spc3.12200

34. Michael Inzlicht and Brandon J. Schmeichel. 2012. What Is Ego Depletion? Toward a Mechanistic Revision of the Resource Model of Self-Control. Perspectives on Psychological Science 7, 5 (2012), 450-463. http://dx.doi.org/10.1177/1745691612454134

35. Yoona Kang, June Gruber, and Jeremy R. Gray. 2012. Mindfulness and de-automatization. Emotion Review 5 , 2 (July 2012), 192-201. http://dx.doi.org/10.1177/1754073912451629
36. Alan Kingstone, Daniel Smilek, Jelena Ristic, Chris Kelland Friesen, and John D. Eastwood. 2003. Attention, researchers! It is time to take a look at the real world. Current Directions in Psychological Science 12, 5 (2003), 176-180. http://dx.doi.org/10.1111/1467-8721.01255

37. Robert Kurzban, Angela Duckworth, Joseph W. Kable, and Justus Myers. 2013. An opportunity cost model of subjective effort and task performance. Behavioral and Brain Sciences 36, 06 (2013), 661-679. http://dx.doi.org/10.1017/s0140525x12003196

38. Edward McAuley, Terry Duncan, and Vance V. Tammen. 1989. Psychometric properties of the Intrinsic Motivation Inventory in a competitive sport setting: A confirmatory factor analysis. Research Quarterly for Exercise and Sport 60, 1 (1989), 48-58. http://dx.doi.org/10.1080/02701367.1989.106074

39. Jane McGonigal. 2009. Super Better - or how to turn recovery into a multi-player experience. Avant Game. Retrieved from http://blog.avantgame.com/2009/09/super-better-orhow-to-turn-recovery.html

40. Monica Melby-Lervåg and Charles Hulme. 2013. Is working memory training effective? A meta-analytic review. Developmental Psychology 49, 2 (2013), 270291.

http://dx.doi.org/10.1037/a0028228

41. Monica Melby-Lervag, Thomas S. Redick, and Charles Hulme. 2016. Working memory training does not improve performance on measures of intelligence or other measures of "far transfer": Evidence from a metaanalytic review. Perspectives on Psychological Science 11, 4 (January 2016), 512-534. http://dx.doi.org/10.1177/1745691616635612

42. Monica Melby-Lervag, Thomas S. Redick, and Charles Hulme. 2016. Working memory training does not improve performance on measures of intelligence or other measures of "far transfer": Evidence from a metaanalytic review. Perspectives on Psychological Science 11, 4 (January 2016), 512-534. http://dx.doi.org/10.1177/1745691616635612

43. Eleanor Miles, Paschal Sheeran, Harriet Baird, Ian Macdonald, Thomas L. Webb, and Peter R. Harris. 2016. Does self-control improve with practice? Evidence from a six-week training program. Journal of Experimental Psychology: General 145, 8 (2016), 1075-1091. http://dx.doi.org/10.1037/xge0000185

44. Marina Milyavskaya and Michael Inzlicht. 2017. What's So Great About Self-Control? Examining the Importance of Effortful Self-Control and Temptation in Predicting Real-Life Depletion and Goal Attainment. Social Psychological and Personality Science (2017), 194855061667923. http://dx.doi.org/10.1177/1948550616679237 
45. Walter Mischel, Shoda Yuichi, and Monica L. Rodriguez. 1989. Delay of gratification in children.

Science 244, 4907 (1989), 933-938.

http://dx.doi.org/10.1126/science.2658056

46. Akira Miyake, Naomi P. Friedman, Michael J. Emerson, Alexander H. Witzki, Amy Howerter, and Tor D. Wager. 2000. The Unity and Diversity of Executive Functions and Their Contributions to Complex "Frontal Lobe" Tasks: A Latent Variable Analysis. Cognitive Psychology 41, 1 (2000), 49-100. http://dx.doi.org/10.1006/cogp.1999.0734

47. Terrie E. Moffitt et al. 2011. A gradient of childhood self-control predicts health, wealth, and public safety. Proceedings of the National Academy of Sciences 108, 7 (2011), 2693-2698. http://dx.doi.org/10.1073/pnas.1010076108

48. Daniel C. Molden et al. 2012. Motivational Versus Metabolic Effects of Carbohydrates on Self-Control. Psychological Science 23, 10 (2012), 1137-1144. http://dx.doi.org/10.1177/0956797612439069

49. Mark Muraven. 2010. Building self-control strength: Practicing self-control leads to improved self-control performance. Journal of Experimental Social Psychology 46, 2 (2010), 465-468. http://dx.doi.org/10.1016/j.jesp.2009.12.011

50. Mark Muraven, Dianne M. Tice, and Roy F. Baumeister. 1998. Self-control as a limited resource: Regulatory depletion patterns. Journal of Personality and Social Psychology 74, 3 (1998), 774-789.

http://dx.doi.org/10.1037/0022-3514.74.3.774

51. Kristian Ove R. Myrseth and Ayelet Fishbach. 2009. Self-Control: Self-control a function of knowing when and how to exercise restraint. Current Directions in Psychological Science 18, 4 (2009), 247-252. http://dx.doi.org/10.1111/j.1467-8721.2009.01645.x

52. Pier J. Prins, Sebastiaan Dovis, Albert Ponsioen, Esther Ten Brink, and Saskia Van Der Oord. 2011. Does computerized working memory training with game elements enhance motivation and training efficacy in children with ADHD? Cyberpsychology, Behavior, and Social Networking 14, 3 (2011), 115-122. http://dx.doi.org/10.1089/cyber.2009.0206

53. Andrew K. Przybylski, C. Scott Rigby, and Richard M. Ryan. 2010. A motivational model of video game engagement. Review of General Psychology 14, 2 (2010), 154-166. http://dx.doi.org/10.1037/a0019440

54. Dorothy Rethlingshafer. 1942. Relationship of tests of persistence to other measures of continuance of activities. The Journal of Abnormal and Social Psychology 37, 1 (1942), 71-82.

http://dx.doi.org/10.1037/h0056753

55. Richard M. Ryan and Edward L. Deci. 2000. Selfdetermination theory and the facilitation of intrinsic motivation, social development, and wellbeing. American Psychologist 55, 1 (2000), 68-78. http://dx.doi.org/10.1037//0003-066x.55.1.68

56. Richard M. Ryan, C.Scott Rigby, and Andrew Przybylski. 2006. The Motivational Pull of Video Games: A Self-Determination Theory Approach. Motivation and Emotion Motiv Emot 30, 4 (2006), 344-360. http://dx.doi.org/10.1007/s11031-006-9051-8

57. Katie Salen and Eric Zimmerman. 2003. Rules of Play: Game Design Fundamentals. MIT Press 2004: 688. https://doi.org/10.1093/intimm/dxs150

58. Carol Sansone, Charlene Weir, Lora Harpster, and Carolyn Morgan. 1992. Once a boring task always a boring task? Interest as a self-regulatory mechanism. Journal of Personality and Social Psychology 63, 3 (1992), 379-390. http://dx.doi.org/10.1037/00223514.63.3.379

59. Fleming Seay and Robert E. Kraut. 2007. Project Massive: Self-Regulation and Problematic Use of Online Gaming. Proceedings of the SIGCHI conference on Human factors in computing systems CHI '07. https://doi.org/10.1145/1240624.1240749

60. Shauna L. Shapiro, Linda E. Carlson, John A. Astin, and Benedict Freedman. 2006. Mechanisms of mindfulness. Journal of Clinical Psychology. 62, 3 (2006), 373-386. http://dx.doi.org/10.1002/jclp.20237

61. Milad Soroush, Mark Hancock, and Vanessa K. Bohns. 2014. Self-control in casual games: The relationship between Candy Crush Saga TM players' in-app purchases and self-control. In Conference Proceedings - 2014 IEEE Games, Media, Entertainment Conference, IEEE GEM (2014). https://doi.org/10.1109/GEM.2014.7048099

62. Yi-Yuan Tang et al. 2007. Short-term meditation training improves attention and self-regulation. Proceedings of the National Academy of Sciences 104, 43 (November 2007), 17152-17156. http://dx.doi.org/10.1073/pnas.0707678104

63. June P. Tangney, Roy F. Baumeister, and Angie L. Boone. 2004. High self-control predicts good adjustment, less pathology, better grades, and interpersonal success. Journal of Personality 72, 2 (2004), 271-324. http://dx.doi.org/10.1111/j.0022-3506.2004.00263.x

64. George R. Thornton. 1939. A factor analysis of tests designed to measure persistence. Psychological Monographs 51, 3 (1939), i-42. http://dx.doi.org/10.1037/h0093472

65. David Watson, Lee A. Clark, and Auke Tellegen. 1988. Development and validation of brief measures of positive and negative affect: The PANAS scales. Journal of Personality and Social Psychology 54, 6 (1988), 10631070. http://dx.doi.org/10.1037/0022-3514.54.6.1063 
66. Georgios N. Yannakakis and John Hallam. 2009. RealTime Game Adaptation for Optimizing Player Satisfaction. IEEE Trans. Comput. Intell. AI Games IEEE Transactions on Computational Intelligence and AI in Games 1, 2 (2009), 121-133.

http://dx.doi.org/10.1109/tciaig.2009.2024533

67. About SupperBetter - SupperBetter. Retrieved August 8, 2018 from https://www.superbetter.com/about

68. Habitica - Gamify Your Life. Retrieved August 8, 2018 from https://habitica.com/static/home

69. A Consensus on the Brain Training Industry from the Scientific Community. 2014. Retrieved September 21,
2016 from

http://longevity3.stanford.edu/blog/2014/10/15/theconsensus-on-the-brain-training-industry-from-thescientific-community-2/

70. Lumosity devs pay FTC $\$ 2 \mathrm{M}$ over deceptive brain game advertising. Retrieved September 21, 2016 from https://www.ftc.gov/news-events/pressreleases/2016/01/lumosity-pay-2-million-settle-ftcdeceptive-advertising-charges 